\title{
Cancer/Testis Antigen MAGE-C1/CT7: New Target for Multiple Myeloma Therapy
}

\author{
Fabricio de Carvalho, ${ }^{1}$ André L. Vettore, ${ }^{2}$ and Gisele W. B. Colleoni ${ }^{1}$ \\ ${ }^{1}$ Disciplina de Hematologia e Hemoterapia, Universidade Federal de São Paulo, UNIFESP/EPM, Rua Botucatu, \\ 04023-900 Vila Clementino, SP, Brazil \\ ${ }^{2}$ Departamento de Ciências Biológicas, UNIFESP, 09972-270, Diadema, SP, Brazil
}

Correspondence should be addressed to Fabricio de Carvalho, fcarvalho@unifesp.br

Received 4 November 2011; Accepted 28 December 2011

Academic Editor: Mohamad Mohty

Copyright (C) 2012 Fabricio de Carvalho et al. This is an open access article distributed under the Creative Commons Attribution License, which permits unrestricted use, distribution, and reproduction in any medium, provided the original work is properly cited.

Cancer/Testis Antigens (CTAs) are a promising class of tumor antigens that have a limited expression in somatic tissues (testis, ovary, fetal, and placental cells). Aberrant expression of CTAs in cancer cells may lead to abnormal chromosome segregation and aneuploidy. CTAs are regulated by epigenetic mechanisms (DNA methylation and acetylation of histones) and are attractive targets for immunotherapy in cancer because the gonads are immune privileged organs and anti-CTA immune response can be tumor-specific. Multiple myeloma (MM) is an incurable hematological malignancy, and several CTAs have been detected in many MM cell lines and patients. Among CTAs expressed in MM we must highlight the MAGE-C1/CT7 located on the X chromosome and expressed specificity in the malignant plasma cells. MAGE-C1/CT7 seems to be related to disease progression and functional studies suggests that this CTA might play a role in cell cycle and mainly in survival of malignant plasma cells, protecting myeloma cells against spontaneous as well as drug-induced apoptosis.

\section{Cancer/Testis Antigens}

Tumors generally are immunogenic. They produce proteins that normally are not expressed in tissues from adults and therefore are not considered self by the immune system [1].

The idea that immune system can recognize and respond to these tumor proteins (antigens) was postulated at the end of the 19th century, when William Coley, a surgeon at Memorial Sloan-Kettering Cancer Center in New York (USA), observed that rare events of spontaneous tumor progression were often preceded by infectious episodes [2].

Tumor immunology began several decades ago, when it was shown that mice could be immunized against syngeneic tumors and that the antibodies produced led to specific rejection of transplanted tumor tissue $[3,4]$. Thomas and Burnet introduced the concept of cancer immune surveillance to describe a mechanism of protection against tumors in immunocompetent hosts. From this, the development of tumor vaccines for the human population became a new possibility for cancer treatment [2].
Tumor-associated antigens were originally discovered in patients with malignant melanoma. These antigens were subsequently identified in several types of human tumors. In normal tissue, they were first described in testicular germ line. Therefore, genes that express these proteins were called cancer/testis antigens (CTAs) [5-7].

Thus, the CTAs are a promising class of tumor antigens due to its limited expression in somatic tissues (germ cells of the testis, ovary, fetal, and placental cells (trophoblast)) [8-10]. Some CTAs can be expressed in other normal tissues such as pancreas, liver, and spleen, but the level of expression is much smaller than observed in germ cells [9].

The study by van der Bruggen et al. [11] was the first to demonstrate that CTAs could be specific recognized in vitro by cytotoxic $\mathrm{T}$ lymphocytes (CTLs) in patients with melanoma. It was also possible to obtain autologous antitumor CTL by cultures of irradiated tumor cells with blood lymphocytes of melanoma-bearing patient $[12,13]$.

Testicular and placental cells did not express MHC (Major Histocompatibility Complex) class I, and the CTAs are 
not recognized by CD8+ cytotoxic T lymphocytes. For this reason, CTAs are considered excellent targets for antitumor cell vaccines [14].

The development of vaccines for specific-tumor antigens depends in part on the identification of a broad spectrum of immunogenic proteins expressed predominantly in human cancer. The technique of cloning T-cell epitopes, developed and published in 1991, led to the discovery of the human CTAs MAGEA1, BAGE, and GAGE. It was demonstrated that the products of the mRNAs transcribed and translated these CTAs almost exclusively in normal testis and various tumor types [15].

In 1995, the SEREX (Serological Analysis of cDNA Expression Libraries) technique was used in the search of new tumor antigens recognized by IgG of cancer patients [16]. Among the genes identified by SEREX in human tumors were SSX2, NY-ESO-1, and SYCP-1, which were also, predominantly, expressed in normal testis and cancer [15].

Scanlan et al. [15] classify the CTAs into four categories, according to the expression profile measured qualitatively by conventional RT-PCR (Reverse Transcriptase-Polymerase Chain Reaction): (1) CTAs exclusively expressed in testis and tumors (Testis-Restricted), (2) CTAs expressed in two or more nongametogenic tissues (Tissue-Restricted), (3) CTAs expressed in 3-6 nongametogenic tissues (Differentially Expressed), and (4) CTAs expressed in $>6$ nongametogenic tissues (Ubiquitously Expressed) [15, 17, 18].

With the description of an increasing number of CTAs, it was necessary to implement a nomenclature to differentiate these genes. In general, due to lack of information about the CTA functions in cellular environment, the nomenclature was based on the chronological order of discovery (e.g., MAGEA is CT1; BAGE is CT2). There are cases of multiple members in CTA families; in those cases, each member of a family is assigned as a number, for example, SSX1 is CT5.1, SSX2 is CT5.2, SSX3 is CT5.3, and so on [15].

In 2008, a new classification of CTAs was developed from in silico analysis of cDNA Database (http://evocontology .org), MPSS (Massively Parallel Signature Sequencing), CAGE (Cap Analysis Gene-Expression) and data expression obtained by conventional RT-PCR. In this classification, the CTAs were divided basically into three distinct groups: Testis-Restricted (CTAs expressed in normal adult testis and placenta), Testis/Brain-Restricted (CTAs expressed in normal adult testis and in all brain tissues), and Testis-Selective (CTAs were classified according to the ratio between the expression of normal adult testis/placenta in relation to others expressed in normal adult tissues) [6].

More than 250 CTAs were described in the CT antigen database (http://www.cta.lncc.br). The CTAs can be divided between those who are located on the $\mathrm{X}$ chromosome and those which are present in other chromosomes (the autosomes) $[19,20]$. CTAs located on the X chromosome, such as $M A G E-C 1 / C T 7$ gene, tend to form families that are normally expressed in spermatogonia in a coordinated manner [21, 22] (Figure 1). The careful annotation of the genes present on the X chromosome showed that approximately $10 \%$ of

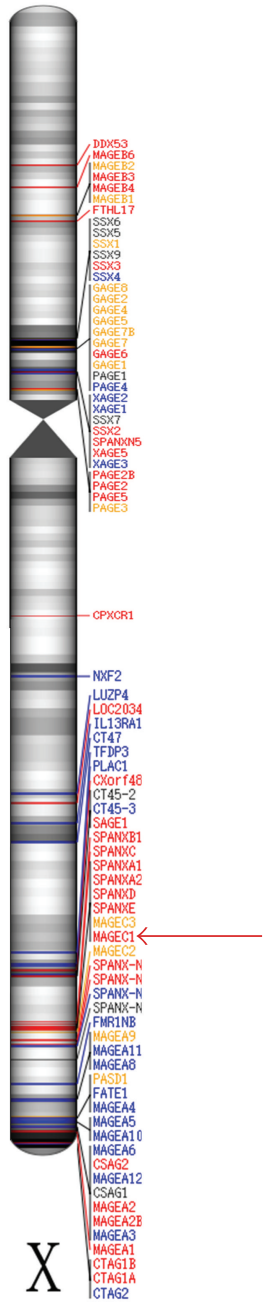

- CTAs Testis-Restricted

CTAs Testis/Brain-Restricted

$\Delta$ CTAs Testis-Selective

CTAs not found

FIGURE 1: Distribution of cancer/testis antigens (CTAs) on the X chromosome. Black regions on chromosome demonstrate a high density of ESTs (Expressed Sequence Tags). Red Arrow indicates CTA MAGE-C1/CT7 (Testis-Restricted) (Font: Hofmann et al. [6]; modified by de Carvalho et al. 2011).

them are CTAs and are often coexpressed in tumor cells $[19,22,23]$.

In the testis, CTAs are typically expressed in spermatocytes and act in meiosis. Thus, the aberrant expression of CTAs in cancer cells may lead to abnormal chromosome segregation and aneuploidy, justifying its importance in tumorigenesis $[22,24]$.

CTAs expression is regulated by epigenetic mechanisms such as hypermethylation of the promoter region of the genes (DNA methylation) and acetylation of histones [9, 19, 20, 22]. Therefore, since CTAs are not expressed or have low expression in differentiated somatic tissues, some authors suggest that the expression of CTAs in tumor tissue 
may be restricted to cells that retain stem cell properties $[9,25]$. In tumors, a restricted population with stem cell properties (cancer stem cells) can favor tumor maintenance, proliferation, and metastasis [9].

The MAGE genes may be involved in tumor transformation or in some aspects of tumor progression such as in tumor metastasis [11]. The MAGE genes are frequently expressed in human tumors of different histological types but not expressed in normal tissues except in male germ cells. The CTAs encoded by MAGE genes are recognized by CTL and are strictly tumor-specific [13].

Thus, all CTAs are in principle attractive targets for immunotherapy in cancer because the gonads are immune privileged organs and anti-CTA immune response can be tumor-specific. Vaccines using peptides derived from NYESO-1 (CTAG-1B) have shown clinical benefits in patients with melanoma $[26,27]$.

\section{CTAs Expression in Multiple Myeloma}

Multiple myeloma (MM) is a hematological malignancy secondary to clonal expansion of plasma cells, characterized by the presence of monoclonal immunoglobulin in blood and/or urine, lytic bone lesions, and infiltration of monoclonal plasma cells in bone marrow [28-30].

MM corresponds to $1 \%$ of all malignancies and 10 $15 \%$ of hematologic malignancies and it is the second most common type of blood cancer [14, 31-34]. In the United States, 20,000 new cases of MM are diagnosed every year, with about 11,000 deaths by this disease in the same period of time [33, 35].

The diagnosis of MM is based on the presence of monoclonal protein (M protein) in serum and/or urine, bone marrow infiltration by at least $10 \%$ of clonal plasma cells, and damage to one or more target organs (CRAB: hypercalcemia, renal failure, anemia, bone lesions). Individuals with multiple myeloma must be distinguished from those with Monoclonal Gammopathy of Undetermined Significance (MGUS) $(<10 \%$ plasma cells in bone marrow, low levels of M-protein ( $<3 \mathrm{~g} / \mathrm{dL})$, and no osteolytic lesion), amyloidosis, or other lymphoproliferative disorders with paraproteinemia [36].

The characterization of the mechanisms responsible for the expansion of MM tumor cells is difficult and involves a series of genetic alterations and changes in the bone marrow microenvironment, promoting tumor growth and the failure of the immune system to recognize it $[28,36]$.

Regardless of prognostic factors, MM remains incurable with median overall survival of 3-5 years [32, 35, 37-40]. Although it is possible to obtain complete remission of disease in approximately $25-50 \%$ of patients with initial diagnosis (treated with high-dose melphalan and autologous hematopoietic stem cell transplant), almost all will relapse within 2-3 years [18], suggesting that an effective maintenance therapy is needed to control or slow the progression of the disease [37, 41].

Evidences suggest that small fractions of MM cells escape the action of chemotherapy and remains undetectable by conventional methods, explaining the recurrence of the disease. These small fractions of myeloma cells are considered potential targets for immunotherapy, either active or passive, due to two main factors: (1) small fractions of MM cells can be destroyed by cytotoxic T lymphocytes, and (2) in allogeneic hematopoietic stem cell transplants, infusion of donor $\mathrm{T}$ cells can efficiently eliminate the small fraction of residual MM cells [18].

The vaccines formulated with antigens associated with MM can instruct the immune system to eliminate malignant cells. But specific antigens of myeloma cells are required [14].

The immunoglobulin (Ig) clones that are produced by small fractions of residual MM cells are considered ideal targets for building and specific anti-idiotype vaccines. However, several clinical studies using anti-idiotype vaccines targeting the Ig failed to demonstrate benefit in patients with MM [18].

CTAs have been detected in many cell lines and primary tumor samples from patients with MM by RT-PCR and immunohistochemistry [14]. Despite the little information available about their importance as clinical prognostic factors or related to aberrant proliferation of malignant plasma cells, some studies with different tumor cell lines have shown an association between the expression of CTAs and a phenotype of resistance to chemotherapy treatments $[42,43]$.

There are evidences that CTAs are also expressed in relapsed MM samples and may be considered important prognostic markers in newly diagnosed MM patients and in relapsed cases [44]. On the other hand, the expression of CTAs in many hematologic malignancies such as leukemia and B-cell non-Hodgkin's lymphomas is considered a rare event [45].

The expression of CTA members of the MAGE family in tumor cells appears to contribute directly to the malignant phenotype and poor response to therapy [22, 46, 47]. MAGE family members are present on the $\mathrm{X}$ chromosome and are described as CTA-X-MAGE. Moreover, all members of this family present a 200 amino acids common domain, known as MHD (MAGE Homology Domain), involved in proteinprotein interactions $[48,49]$.

In our previous study, Andrade et al. [50] showed that three CTAs (localized on the X chromosome) MAGEC1/CT7, MAGE-A3/6, and LAGE-1 were often expressed in $\mathrm{MM}$ suggesting that they could be good candidates for immunotherapy. According this study, CTA MAGE-C1/CT7 gene was the most frequently expressed CTA in MM and seems to have prognostic impact in overall survival.

\section{MAGE-C1/CT7: New Target for Immunotherapy in Myeloma}

Tumor-specific immunotherapy is a promising strategy for treating patients with $\mathrm{MM}$, but a T-cell-based therapy depends on identification of an antigen expressed strictly in tumor cells. Myeloma is a tumor of B cells and therefore has the potential to present antigen directly to $\mathrm{T}$ cells, although the ability to present the malignant plasma cells is believed 


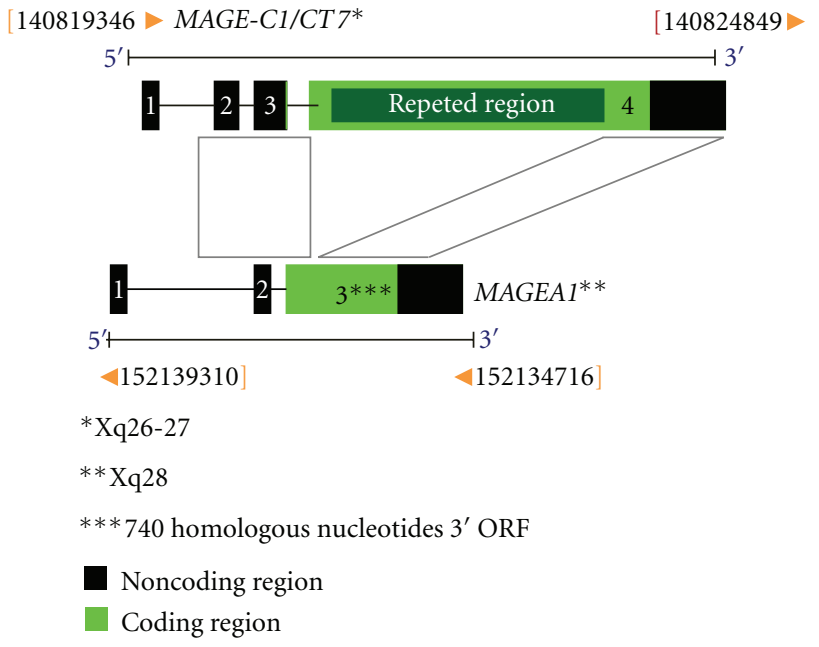

FIGURE 2: Schematic representation of MAGE-C1/CT7 gene: comparison of content structure MAGE-C1/CT7 and MAGEA1 genes. The ORFs (Open Reading Frames) are indicated in green. The black regions are exons. Repeated region of the MAGE-C1/CT7 gene is highlighted in exon 4 . The gray areas show regions of homology between the two CTAs (Font: Lucas et al. [53]; modified by de Carvalho et al. 2011).

to be limited. The antigen presentation of CTA proteins can thus arise from cross-priming by dendritic cells. Due to MAGE-C1/CT7 antigen expression in restricted in tumor cells, it seems to be a promising candidate for immunotherapy in MM [51].

The CT7 gene is located in the region Xq26-27 (Figure 1) and was identified by SEREX in the melanoma cell line SK-MEL-37 and allogeneic serum of melanoma patients [52]. The CT7 gene is identical to MAGE-C1, identified by RDA (Representational Difference Analysis) [53, 54]. Near the region of $M A G E-C 1 / C T 7$ are two subfamilies that are MAGE-B (composed of four genes that are located in the region Xq21.3) and MAGE-A (with 12 members located in the region $\mathrm{Xq} 28$ ). MAGE-C1/CT7 represents the first member of a new subfamily [53].

In evolutionary terms, MAGE-C1/CT7 gene is considered recent, present in primates (chimpanzee and rhesus) and humans. MAGE-C1/CT7 protein has a region homologous to the MAGE family, corresponding to 275 amino acids of the carboxyl-terminal region. In the amino-terminal region, a segment composed of tandem repeats presents several SNPs (Single Nucleotide Polymorphisms) (Figure 2). The most common amino acids in this repeated region are serine, proline, and glutamine, representing $53 \%$ of the whole sequence. MAGE-C1/CT7 is unique compared to MAGE families' proteins because the repeated region of this CTA has a distinct conformation compared to the other MAGE proteins [52, 53]. Lucas et al. [53] demonstrated that the repeated region presented in the amino-terminal of MAGEC1/CT7 protein ranged from 667 to 1052 amino acids, resulting in the identification of six distinct alleles.

MAGE-C1/CT7 is considered a testis-restricted CTA by the current classification [6], because it is only expressed in normal adult testis and several studies have shown that this CTA is expressed in a wide variety of human tumors $[6,53$, 55].

Cho et al. [19] demonstrated that MAGE-C1/CT7 protein is preferably located in the cytoplasm, but it has also been found in the cell nucleus. The same authors suggested that there is physical interaction between MAGE-C1/CT7 and NY-ESO-1 proteins, suggesting that the coordinated expression of two genes is a common event in many types of tumors, including MM [19]. Moreover, the expression of $M A G E-C 1 / C T 7$ in $\mathrm{MM}$ is seen as being restricted to the malignant plasma cells $[14,34]$.

Dhodapkar et al. [56] demonstrated that MAGEC1/CT7 protein was expressed in most samples from MM, medullary plasmacytoma, and extramedullary plasmacytoma by immunohistochemistry. The same authors also observed the expression of MAGE-C1/CT7 antigen on the cell surface of the CAG cell line by flow cytometry and one case of plasmacytoma by immunohistochemistry, suggesting the expression of this CTA on the cell surface myeloma. However this fact should be future investigated because it was the only study to detect the expression of CTA on the cell surface [56].

Jungbluth et al. [14] observed that MAGE-C1/CT7 gene expression is related to disease progression in myeloma due to its high expression in samples of MM stage III, compared to individuals with MGUS. Condomines et al. [18] demonstrated that MAGE-C1/CT7 was more expressed (66\%) in patients with newly diagnosed $\mathrm{MM}$ and in those who survived the treatment. Tinguely et al. [57] showed that the expression of MAGE-C1/CT7 does not correlated with survival of malignant plasma cells but observed that patients with MAGE-C1/CT7 protein located in the cell cytoplasm had a better prognosis than those patients who showed protein expression in the nucleus.

Andrade et al. [50] observed high-frequency (77\%) $M A G E-C 1 / C T 7$ expression in MM patients with advanced stage and possible unfavorable impact on prognosis related to expression of this gene. In the same study, the MAGEC1/CT7 expression was also observed in patients with MGUS (33\%) and bone marrow of patients with solitary plasmacytoma $(20 \%)$.

Curioni-Fontecedro et al. [58], through an immunogenicity study of MAGE-C1/CT7 antigen in vivo, showed that this CTA was responsible for the high frequency of specific IgG antibodies in MM patients. Furthermore, they observed specific immune response against the MAGEC1/CT7, demonstrating that antimyeloma immunity can be generated in patients with this disease.

Atanackovic et al. [59], evaluating the prognostic value of MAGE-C1/CT7 expression in MM, demonstrated that patients undergoing allogeneic hematopoietic stem cells transplant showed early recurrence and worse overall survival when the malignant plasma cells from bone marrow expressed MAGE-C1/CT7. The same authors suggest that the CTAs in general are involved in the progression of myeloma, further increasing the aggressiveness of the tumor and that the MAGE-C1/CT7 might be considered as a gatekeeper gene for other CTAs [59]. 
Nuber et al. [55] identified and characterized naturally occurring MAGE-C1/CT7-specific T lymphocytes in patients with melanoma expressing this CTA, suggesting a strong immunogenicity of this antigen and that MAGE-C1/CT7 could be a good candidate for immunotherapy. Lendvai et al. [60] demonstrated the presence of T lymphocytes specific for MAGE-C1/CT7 in patients with MM.

Anderson et al. [61] identified immunogenic CD8+ Tcell epitopes of MAGE-C1/CT7 and demonstrated that these epitopes are naturally processed and presented by tumor cells.

Atanackovic et al. [45] throughout transient silencing of MAGE-C1/CT7 and MAGE-A3 genes suggested that both CTAs are involved in the survival of myeloma cells, decreasing apoptosis induced by chemotherapy. The same authors also demonstrated that transient silencing of MAGE-C1/CT7 in $\mathrm{MM}$ cell lines affected the MAGE-C2/CT10 expression, indicating a possible interaction between both genes [45].

Recently, our group (de Carvalho et al.) [24] throughout stable silencing of MAGE-C1/CT7 by shRNA (Short Hairpin $R N A$ ) in MM cell lines showed that MAGE-C1/CT7 is involved in survival of malignant plasma cells, protecting myeloma cells against spontaneous as well as drug-induced (bortezomib-inhibitor of the $26 \mathrm{~S}$ ubiquitin/proteasome) apoptosis. We also suggest that this CTA might play a role in cell cycle and speculate that silencing MAGE-C1/CT7 might represent a valuable therapeutic option for $\mathrm{MM}$, in particular when applied in combination with proteasome inhibitors. However, the exact function of MAGE-C1/CT7 protein in the pathophysiology of MM is not yet understood [24].

\section{Concluding Remarks}

CTAs are attractive targets for immunotherapy in cancer due to its limited expression in somatic tissues. These antigens have been detected in MM patients and might be used for T-cell immunotherapy. However, we still know little about the actual role of CTAs in the biology of this incurable disease. Several studies demonstrate that the MAGE-C1/CT7 is commonly expressed in $\mathrm{MM}$ and has important role in the development and prognosis of the disease, making it a possible therapeutic target. Nevertheless, further in vitro, in vivo and clinical studies should be conducted in order to better understand the participation of MAGE-C1/CT7 and other CTAs in the MM tumorigenesis and to clarify the biological pathways in which these proteins act.

\section{References}

[1] J. Bangham, "Auspicious antigens," Nature Reviews Cancer, vol. 5, article 499, 2005.

[2] E. Gilboa, "The promise of cancer vaccines," Nature Reviews Cancer, vol. 4, no. 5, pp. 401-411, 2004.

[3] R. T. Prehn and J. M. Main, "Immunity to methylcholanthrene-induced sarcomas," Journal of the National Cancer Institute, vol. 18, no. 6, pp. 769-778, 1957.

[4] G. Klein, H. O. Sjogren, E. Klein, and K. E. Hellstrom, “Dem onstration of resistance against methylcholanthrene-induced sarcomas," Cancer Research, vol. 20, pp. 1561-1572, 1960.
[5] T. Utsunomiya, H. Inoue, F. Tanaka et al., "Expression of cancer-testis antigen (CTA) genes in intrahepatic cholangiocarcinoma," Annals of Surgical Oncology, vol. 11, no. 10, pp. 934-940, 2004.

[6] O. Hofmann, O. L. Caballero, B. J. Stevenson et al., "Genomewide analysis of cancer/testis gene expression," Proceedings of the National Academy of Sciences of the United States of America, vol. 105, no. 51, pp. 20422-20427, 2008.

[7] S. N. Akers, K. Odunsi, and A. R. Karpf, "Regulation of cancer germline antigen gene expression: implications for cancer immunotherapy," Future Oncology, vol. 6, no. 5, pp. 717-732, 2010.

[8] J. R. Peng, H. S. Chen, D. C. Mou et al., "Expression of cancer/testis (CT) antigens in Chinese hepatocellular carcinoma and its correlation with clinical parameters," Cancer Letters, vol. 219, no. 2, pp. 223-232, 2005.

[9] F. F. Costa, K. Le Blanc, and B. Brodin, "Concise review: cancer/testis antigens, stem cells, and cancer," Stem Cells, vol. 25, no. 3, pp. 707-711, 2007.

[10] Y. T. Chen, A. Chadburn, P. Lee et al., "Expression of cancer testis antigen CT45 in classical Hodgkin lymphoma and other B-cell lymphomas," Proceedings of the National Academy of Sciences of the United States of America, vol. 107, no. 7, pp. 3093-3098, 2010.

[11] P. Van Der Bruggen, C. Traversari, P. Chomez et al., "A gene encoding an antigen recognized by cytolytic T lymphocytes on a human melanoma," Science, vol. 254, no. 5038, pp. 16431647, 1991.

[12] C. De Smet, C. Lurquin, E. De Plaen et al., "Genes coding for melanoma antigens recognised by cytolytic T lymphocytes," Eye, vol. 11, no. 2, pp. 243-248, 1997.

[13] P. Chaux, R. Luiten, N. Demotte et al., "Identification of five MAGE-A1 epitopes recognized by cytolytic T lymphocytes obtained by in vitro stimulation with dendritic cells transduced with MAGE-A1," Journal of Immunology, vol. 163, no. 5, pp. 2928-2936, 1999.

[14] A. A. Jungbluth, S. Ely, M. DiLiberto et al., "The cancer-testis antigens CT7 (MAGE-C1) and MAGE-A3/6 are commonly expressed in multiple myeloma and correlate with plasma-cell proliferation," Blood, vol. 106, no. 1, pp. 167-174, 2005.

[15] M. J. Scanlan, A. J. Simpson, and L. J. Old, "The cancer/testis genes: review, standardization, and commentary," Cancer Immunity, vol. 4, article 1, 2004.

[16] K. H. Shain, D. N. Yarde, M. B. Meads et al., " $\beta 1$ integrin adhesion enhances IL-6-mediated STAT3 signaling in myeloma cells: implications for microenvironment influence on tumor survival and proliferation," Cancer Research, vol. 69, no. 3, pp. 1009-1015, 2009.

[17] A. I. Su, T. Wiltshire, S. Batalov et al., "A gene atlas of the mouse and human protein-encoding transcriptomes," Proceedings of the National Academy of Sciences of the United States of America, vol. 101, no. 16, pp. 6062-6067, 2004.

[18] M. Condomines, D. Hose, P. Raynaud et al., "Cancer/testis genes in multiple myeloma: expression patterns and prognosis value determined by microarray analysis," Journal of Immunology, vol. 178, no. 5, pp. 3307-3315, 2007.

[19] H. J. Cho, O. L. Caballero, S. Gnjatic et al., "Physical interaction of two cancer-testis antigens, MAGE-C1 (CT7) and NY-ESO-1 (CT6)," Cancer Immunity, vol. 6, article 12, 2006.

[20] C. A. Glazer, I. M. Smith, M. F. Ochs et al., "Integrative discovery of epigenetically derepressed cancer testis antigens in NSCLC," PLoS One, vol. 4, no. 12, Article ID e8189, 2009. 
[21] O. L. Caballero and Y. T. Chen, "Cancer/testis (CT) antigens: potential targets for immunotherapy," Cancer Science, vol. 100, no. 11, pp. 2014-2021, 2009.

[22] A. J. G. Simpson, O. L. Caballero, A. Jungbluth, Y. T. Chen, and L. J. Old, "Cancer/testis antigens, gametogenesis and cancer," Nature Reviews Cancer, vol. 5, no. 8, pp. 615-625, 2005.

[23] B. J. Stevenson, C. Iseli, S. Panji et al., "Rapid evolution of cancer/testis genes on the X chromosome," BMC Genomics, vol. 8, article 129, 2007.

[24] F. de Carvalho, E. T. Costa, A. A. Camargo et al., "Targeting MAGE-C1/CT7 expression increases cell sensitivity to the proteasome inhibitor bortezomib in multiple myeloma cell lines," PLoS One, vol. 6, no. 11, Article ID e27707, 2011.

[25] R. B. Parmigiani, F. Bettoni, M. D. Vibranovski et al., "Characterization of a cancer/testis (CT) antigen gene family capable of eliciting humoral response in cancer patients," Proceedings of the National Academy of Sciences of the United States of America, vol. 103, no. 48, pp. 18066-18071, 2006.

[26] P. F. Robbins, R. A. Morgan, S. A. Feldman et al., "Tumor regression in patients with metastatic synovial cell sarcoma and melanoma using genetically engineered lymphocytes reactive with NY-ESO-1," Journal of Clinical Oncology, vol. 29, no. 7, pp. 917-924, 2011.

[27] J. Yuan, M. Adamow, B. A. Ginsberg et al., "Integrated NYESO-1 antibody and $\mathrm{CD}^{+}$T-cell responses correlate with clinical benefit in advanced melanoma patients treated with ipilimumab," Proceedings of the National Academy of Sciences of the United States of America, vol. 108, no. 40, pp. 1672316728, 2011.

[28] L. Nadav, B. Z. Katz, S. Baron, N. Cohen, E. Naparstek, and B. Geiger, "The generation and regulation of functional diversity of malignant plasma cells," Cancer Research, vol. 66, no. 17, pp. 8608-8616, 2006.

[29] G. M. Dores, O. Landgren, K. A. McGlynn, R. E. Curtis, M. S. Linet, and S. S. Devesa, "Plasmacytoma of bone, extramedullary plasmacytoma, and multiple myeloma: incidence and survival in the United States, 1992-2004," British Journal of Haematology, vol. 144, no. 1, pp. 86-94, 2009.

[30] R. Fonseca, P. L. Bergsagel, J. Drach et al., "International Myeloma Working Group molecular classification of multiple myeloma: spotlight review," Leukemia, vol. 23, no. 12, pp. 2210-2221, 2009.

[31] Q. B. Li, Z. C. Chen, Y. You, and P. Zou, "Small interfering RNA of cyclooxygenase-2 induces growth inhibition and apoptosis independently of Bcl-2 in human myeloma RPMI8226 cells," Acta Pharmacologica Sinica, vol. 28, no. 7, pp. 1031-1036, 2007.

[32] D. J. White, N. Paul, D. A. Macdonald, R. M. Meyer, and L. E. Shepherd, "Addition of lenalidomide to melphalan in the treatment of newly diagnosed multiple myeloma: the National Cancer Institute of Canada Clinical Trials Group MY. 11 trial," Current Oncology, vol. 14, no. 2, pp. 61-65, 2007.

[33] S. Kumar, "Multiple myeloma-current issues and controversies," Cancer Treatment Reviews, vol. 36, supplement 2, pp. S3S11, 2010.

[34] C. Pabst, J. Zustin, F. Jacobsen et al., "Expression and prognostic relevance of MAGE-C1/CT7 and MAGE-C2/CT10 in osteolytic lesions of patients with multiple myeloma," Experimental and Molecular Pathology, vol. 89, no. 2, pp. 175-181, 2010.

[35] K. C. Anderson, M. Alsina, W. Bensinger et al., "Multiple myeloma," Journal of the National Comprehensive Cancer Network, vol. 9, no. 10, pp. 1146-1183, 2011.
[36] H. Ludwig, "Advances in biology and treatment of multiple myeloma," Annals of Oncology, vol. 16, no. 2, pp. ii106-ii112, 2005.

[37] P. G. Richardson, C. Mitsiades, R. Schlossman, N. Munshi, and K. Anderson, "New drugs for myeloma," Oncologist, vol. 12, no. 6, pp. 664-689, 2007.

[38] R. A. Kyle and S. V. Rajkumar, "Multiple myeloma," Blood, vol. 111, no. 6, pp. 2962-2972, 2008.

[39] A. Broyl, D. Hose, H. Lokhorst et al., "Gene expression profiling for molecular classification of multiple myeloma in newly diagnosed patients," Blood, vol. 116, no. 14, pp. 2543-2553, 2010.

[40] K. Mahtouk, J. Moreaux, D. Hose et al., "Growth factors in multiple myeloma: a comprehensive analysis of their expression in tumor cells and bone marrow environment using Affymetrix microarrays," BMC Cancer, vol. 10, article 198, 2010.

[41] I. M. Ghobrial and A. K. Stewart, "ASH evidence-based guidelines: what is the role of maintenance therapy in the treatment of multiple myeloma?" American Society of Hematology Education Program Book, pp. 587-589, 2009.

[42] Z. Duan, Y. Duan, D. E. Lamendola et al., "Overexpression of MAGE/GAGE genes in paclitaxel/doxorubicinresistant human cancer cell lines," Clinical Cancer Research, vol. 9, no. 7, pp. 2778-2785, 2003.

[43] T. Suzuki, K. Yoshida, Y. Wada et al., "Melanoma-associated antigen-A1 expression predicts resistance to docetaxel and paclitaxel in advanced and recurrent gastric cancer," Oncology Reports, vol. 18, no. 2, pp. 329-336, 2007.

[44] M. van Duin, A. Broyl, Y. de Knegt et al., "Cancer testis antigens in newly diagnosed and relapse multiple myeloma: prognostic markers and potential targets for immunotherapy," Haematologica, vol. 96, no. 11, pp. 1662-1669, 2011.

[45] D. Atanackovic, Y. Hildebrandt, A. Jadczak et al., "Cancertestis antigens MAGE-C1/CT7 and MAGE-A3 promote the survival of multiple myeloma cells," Haematologica, vol. 95, no. 5, pp. 785-793, 2010.

[46] B. Yang, S. M. O’Herrin, J. Wu et al., "MAGE-A, mMage-b, and MAGE-C proteins form complexes with KAP1 and suppress p53-dependent apoptosis in MAGE-positive cell lines," Cancer Research, vol. 67, no. 20, pp. 9954-9962, 2007.

[47] B. Yang, S. O'Herrin, J. Wu et al., "Select cancer testes antigens of the MAGE-A, -B, and -C families are expressed in mast cell lines and promote cell viability in vitro and in vivo," Journal of Investigative Dermatology, vol. 127, no. 2, pp. 267-275, 2007.

[48] P. Chomez, O. De Backer, M. Bertrand, E. De Plaen, T. Boon, and S. Lucas, "An overview of the MAGE gene family with the identification of all human members of the family," Cancer Research, vol. 61, no. 14, pp. 5544-5551, 2001.

[49] P. A. Barker and A. Salehi, "The MAGE proteins: emerging roles in cell cycle progression, apoptosis, and neurogenetic disease," Journal of Neuroscience Research, vol. 67, no. 6, pp. 705-712, 2002.

[50] V. C. Andrade, A. L. Vettore, R. S. Felix et al., "Prognostic impact of cancer/testis antigen expression in advanced stage multiple myeloma patients," Cancer Immunity, vol. 8, article 2, 2008.

[51] O. Goodyear, K. Piper, N. Khan et al., " $\mathrm{CD} 8_{+} \mathrm{T}$ cells specific for cancer germline gene antigens are found in many patients with multiple myeloma, and their frequency correlates with disease burden," Blood, vol. 106, no. 13, pp. 4217-4224, 2005.

[52] Y. T. Chen, A. O. Güre, S. Tsang et al., "Identification of multiple cancer/testis antigens by allogeneic antibody screening of a melanoma cell line library," Proceedings of the National 
Academy of Sciences of the United States of America, vol. 95, no. 12, pp. 6919-6923, 1998.

[53] S. Lucas, C. De Smet, K. C. Arden et al., "Identification of a new MAGE gene with tumor-specific expression by representational difference analysis," Cancer Research, vol. 58, no. 4, pp. 743-752, 1998.

[54] D. A. Chitale, A. A. Jungbluth, D. S. Marshall et al., "Expression of cancer-testis antigens in endometrial carcinomas using a tissue microarray," Modern Pathology, vol. 18, no. 1, pp. 119126, 2005.

[55] N. Nuber, A. Curioni-Fontecedro, C. Mattera et al., "Fine analysis of spontaneous MAGE-C1/CT7—specific immunity in melanoma patients," Proceedings of the National Academy of Sciences of the United States of America, vol. 107, no. 34, pp. 15187-15192, 2010.

[56] M. V. Dhodapkar, K. Osman, J. Teruya-Feldstein et al., "Expression of cancer/testis (CT) antigens MAGE-A1, MAGE-A3, MAGE-A4, CT-7, and NY-ESO-1 in malignant gammopathies is heterogeneous and correlates with site, stage and risk status of disease," Cancer Immun, vol. 3, article 9, 2003.

[57] M. Tinguely, B. Jenni, A. Knights et al., "MAGE-C1/CT-7 expression in plasma cell myeloma: sub-cellular localization impacts on clinical outcome," Cancer Science, vol. 99, no. 4, pp. 720-725, 2008.

[58] A. Curioni-Fontecedro, A. J. Knights, M. Tinguely et al., "MAGE-C1/CT7 is the dominant cancer-testis antigen targeted by humoral immune responses in patients with multiple myeloma," Leukemia, vol. 22, no. 8, pp. 1646-1648, 2008.

[59] A. D. Atanackovic, T. Luetkens, Y. Hildebrandt et al., "Longitudinal analysis and prognostic effect of cancer-testis antigen expression in multiple myeloma," Clinical Cancer Research, vol. 15, no. 4, pp. 1343-1352, 2009.

[60] N. Lendvai, S. Gnjatic, E. Ritter et al., "Cellular immune responses against CT7 (MAGE-C1) and humoral responses against other cancer-testis antigens in multiple myeloma patients," Cancer Immunity, vol. 10, article 4, 2010.

[61] L. D. Anderson Jr., D. R. Cook, T. N. Yamamoto, C. Berger, D. G. Maloney, and S. R. Riddell, "Identification of MAGEC1 (CT-7) epitopes for T-cell therapy of multiple myeloma," Cancer Immunology, Immunotherapy, vol. 60, no. 7, pp. 985997, 2011. 


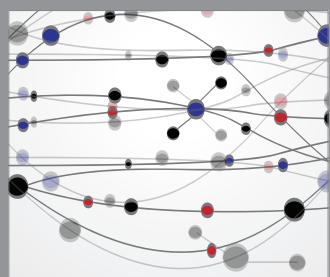

The Scientific World Journal
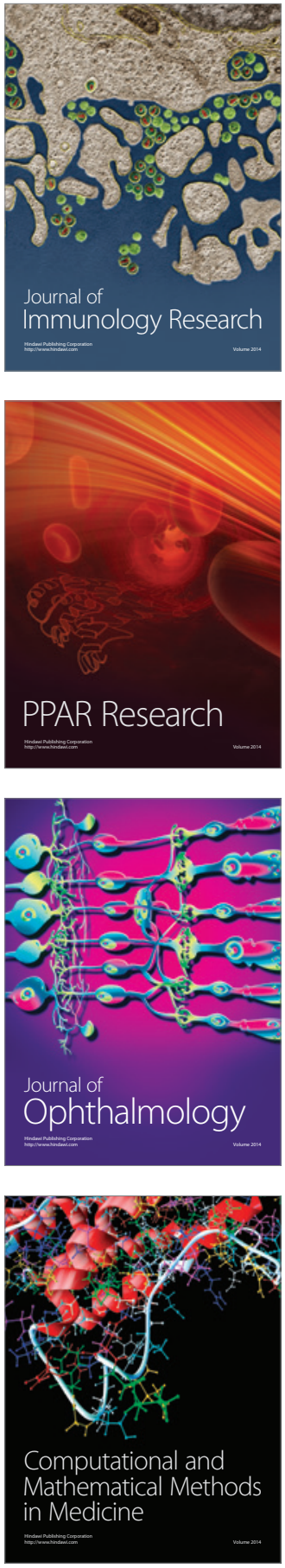

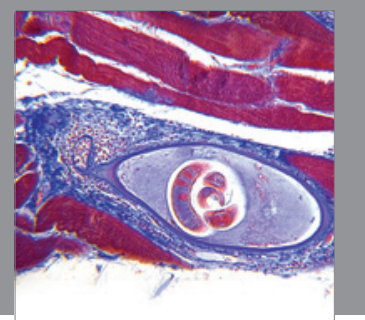

Gastroenterology

Research and Practice
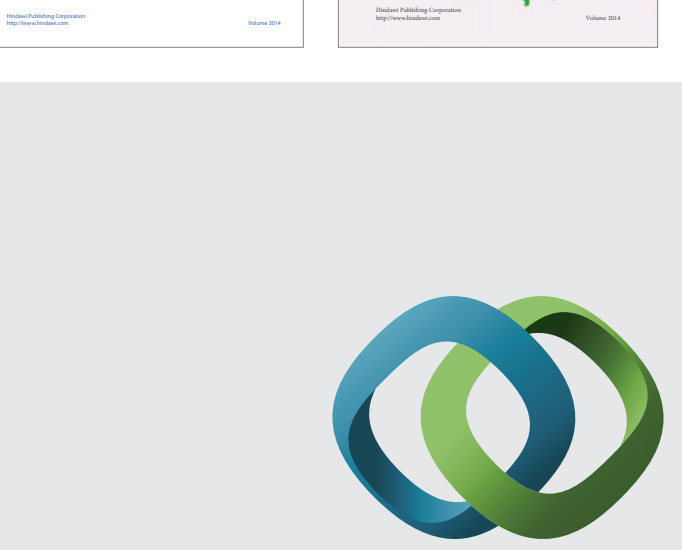

\section{Hindawi}

Submit your manuscripts at

http://www.hindawi.com
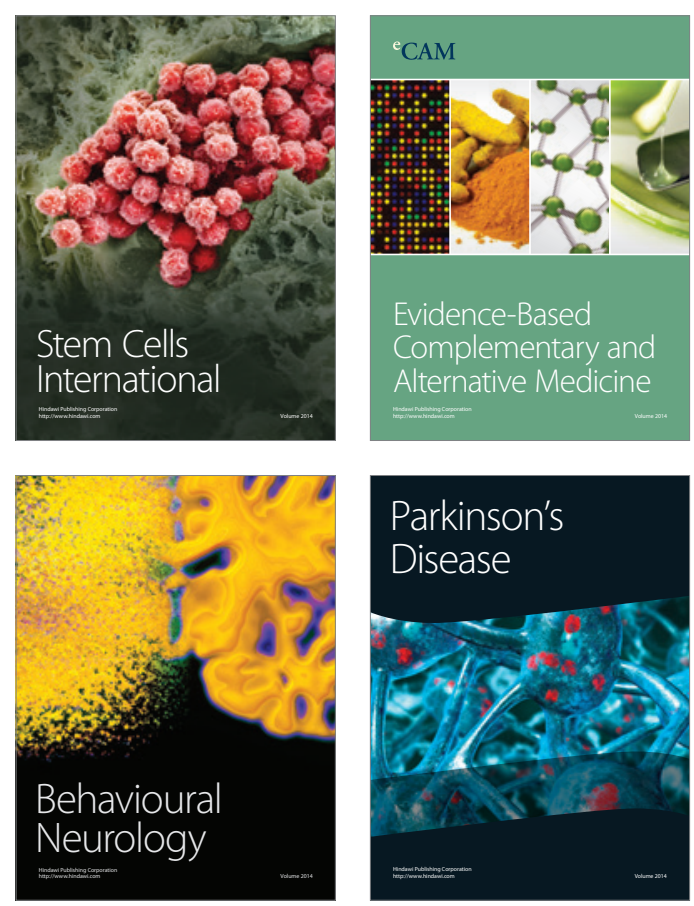

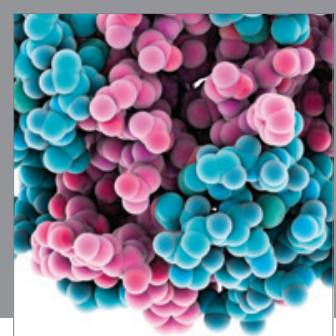

Journal of
Diabetes Research

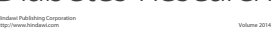

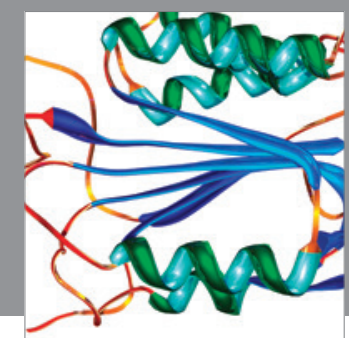

Disease Markers
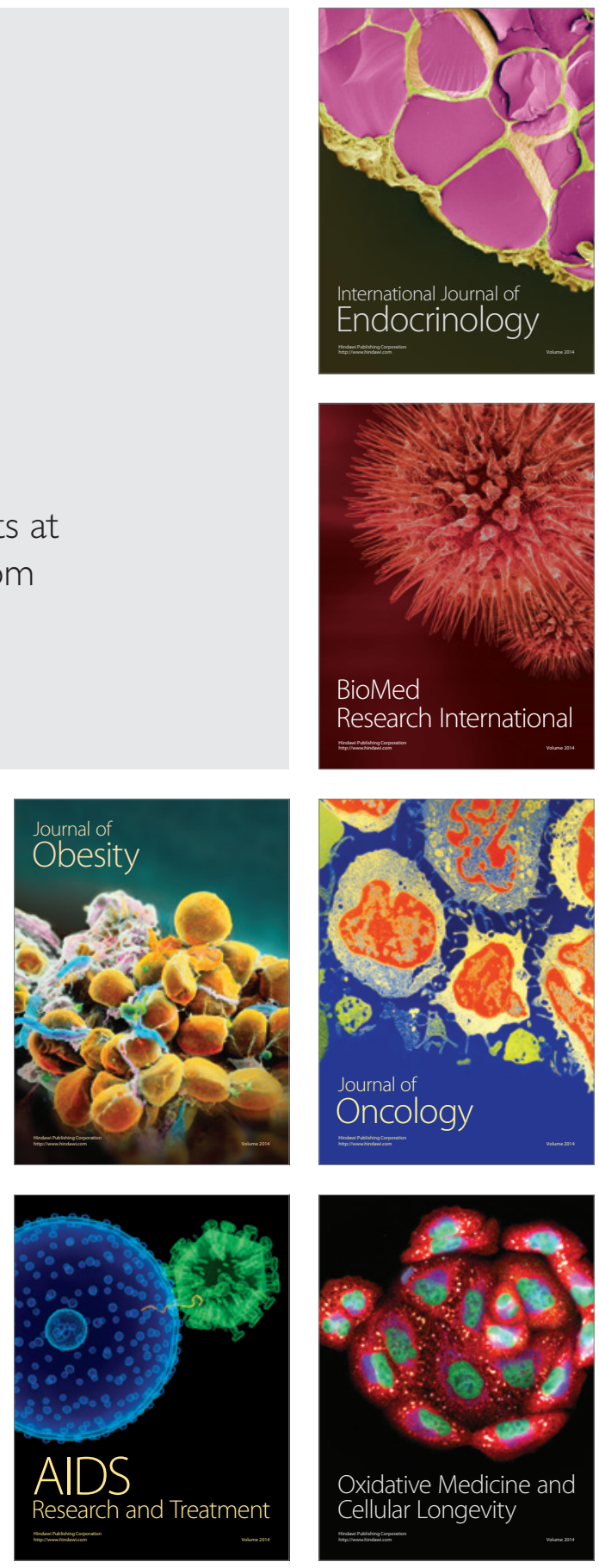